\title{
Cobre no controle da verminose gastrintestinal em ovinos
}

\author{
Copper on the control of gastrintestinal nematodes in sheep
}

\section{Isabel Gonçalves de Gonçalves $^{1}$ Flavio Augusto Menezes Echevarria ${ }^{2}$}

\section{RESUMO}

Este estudo teve o objetivo de verificar o tempo de proteção do cobre contra reinfecções por helmintos gastrintestinais de ovinos em pastejo. Foram utilizados 32 ovinos da raça Corriedale, com aproximadamente 18 meses de idade, mantidos em campo nativo naturalmente contaminado por trichostrongilídeos. Os animais foram pesados e dosificados. Após, foram alocados a quatro grupos experimentais: $T_{I}$ (tratado com cobre e necropsiado no 28ㅁia); $T_{I I}$ (sem cobre e necropsiado no 28o dia); $T_{I I I}$ (tratado com cobre e necropsiado no 56odia); $T_{I V}$ (sem cobre e necropsiado no 56odia). Os grupos $T_{I}$ e $T_{I I I}$ receberam, via oral, uma cápsula gelatinosa de quatro gramas, contendo 3,4 gramas de óxido de cobre. Os ovinos foram avaliados semanalmente através de contagem de ovos por grama de fezes (OPG), volume globular (VG), aspartato aminotransferase (AST) e concentrações de cobre no plasma. Ao abate, foram determinados: carga parasitária, concentrações de cobre no fígado e peso dos fígados. Os resultados demostraram que os níveis de cobre no plasma não tiveram alterações significativas $(P>0,05)$ entre os diversos tratamentos. Quanto aos níveis de cobre detectados no fígado dos animais abatidos observou-se que os lotes medicados com cobre, apresentaram níveis mais elevados que os não medicados $(P<0,05)$, porém dentro dos parâmetros considerados não tóxicos para ovinos. Também foi observado que os níveis de AST, nos grupos tratados com cobre, apresentaram durante todo o período experimental valores levemente superiores aos dos grupos controles, mas diferença significativa $(P<0,05)$ foi detectada apenas entre $T_{I}$ e $T_{I r}$ Quanto à avaliação do $O P G$, não foram observadas diferenças significativas entre os grupos $(P>0,05)$. Com relação aos parasitos gastrintestinais, detectou-se que a administração de cobre contribuiu significativamente para uma menor reinfecção apenas pelo Haemonchus contortus $e$ somente nos animais abatidos na quarta semana após o tratamento, quando a redução foi de $60 \%$ nos medicados $(P<0,05)$. Estima-se que, neste experimento, o cobre não atingiu níveis tóxicos nos animais já que não houve diferença significativa em VG, concentração plasmática de cobre, peso dos fígados e peso vivo $(P>0,05)$. Com base nesses resultados, podemos concluir que 3,4 gramas de óxido de cobre podem ser efetivas na redução das reinfecções por $\boldsymbol{H}$. contortus, durante quatro semanas sem causar toxicidade para ovinos criados extensivamente.

Palavras-chave: cobre, nematódeos, Haemonchus, ovinos, controle.

\section{ABSTRACT}

The aim of this work was to verify the possible activity of copper oxide wire particles against reinfection by gastrintestinal nematodes in sheep under extensive grazing. Thirty-two sheep, all wethers, aged 18 months and kept on native pastures naturally contaminated by trichostrongylides were used. The animals were weighed and treated with anthelmintics and then allocated to four experimental groups: $T_{I}$ (treated with copper and necropsied on Day 28), $T_{H}$ (untreated control and necropsied on Day 28), $T_{I I I}$ (treated with copper and necropsied on Day 56), $T_{I V}$ (untreated control and necropsied on Day 56). Groups $T_{I}$ e $T_{I I I}$ received, per os, a $4 \mathrm{~g}$ bolus containing $3.4 \mathrm{~g}$ of copper oxide wire particles. The animals were monitored, at weekly intervals, for faecal egg counts $(E P G)$, packed cell volume $(P C V)$, aspartate aminotransferase (AST) and plasma copper concentration. At necropsy, worm burdens, liver copper concentration and liver weights were determined. There were no differences in plasma copper levels between treatments $(P>0.05)$. As for copper in the livers, all treated groups had higher levels than the untreated animals $(P<0.05)$. Plasma AST activity was slightly higher during the experimental period in the treated groups. However, significant difference was only detected between treatments $T_{I}$ e $T_{I I}(P<0.05)$. There were no significant differences in EPG counts between treated and control groups $(P>0.05)$. Worm counting at necropsy revealed that copper was effective only against Haemonchus contortus and only in

${ }^{1}$ Médico Veterinário, do Programa de Pós-graduação em Ciências Veterinárias, Universidade Federal do Rio Grande do Sul (UFRGS), Porto Alegre, RS.

${ }^{2}$ Médico Veterinário, MSc, PhD, Laboratório de Helmintologia, Embrapa Pecuária Sul, 96401-970, Bagé, RS. E-mail: echevarr@cppsul.embrapa.br. Autor para correspondência. 
the animals slaughtered on Day $28^{\circ}$ where the reduction was $60 \%$ in comparison to the untreated group $(P<0.05)$. It is believed that in this experiment copper did not reach toxic levels as there were no significant differences in PCV, plasma copper concentrations, liver weights and body weights $(P>0.05)$. It is concluded that $3.4 \mathrm{~g}$ of copper oxide wire particles can have a significant action in reducing reinfections by $\boldsymbol{H}$. contortus during four weeks without toxicity to animals under conditions of extensive grazing.

Key words: copper, nematode, Haemonchus, sheep, control.

\section{INTRODUÇÃO}

O Brasil possui uma população ovina de 16 a 17 milhões de animais, e 55\% destes estão na Região Sul, no Rio Grande do Sul (RS) ondese concentram 95\% dos ovinos lanados, sendo estes criados quase que exclusivamente em pastagem nativa e em associação com gado de corte (ECHEVARRIA, 1996a). $\mathrm{O}$ controle da verminose dos ruminantes baseia-se, principalmente no uso de anti-helmínticos e em alguns casos associado ao manejo das pastagens (PADILHA \& MENDONZA, 1996). Já o controle integrado da verminose ovina (ECHEVARRIA, 1996b) preconiza o pastejo exclusivo com bovinos adultos por um período de quatro meses para redução do número de larvas infectantes; os cordeiros desmamados que entram nessas áreas devem receber duas medicações estratégicas com anti-helmíntico de largo espectro: uma ao adentrarem, e a segunda 60 dias após, além de serem, a partir deste momento, manejados com bovinos adultos. A partir desta segunda medicação, os animais devem ser monitorados através de exames parasitológicos.

No RS, a prevalência de resistência antihelmíntica nos rebanhos ovinos representa um grave problema para o controle eficiente das helmintoses gastrintestinais (ECHEVARRIA et al., 1996). A resistência anti-helmíntica em nematódeos de ovinos se constitui no principal problema sanitário com que se defronta a indústria ovinícola no Brasil (ECHEVARRIA, 1996b). Esta situação não é diferente em outros países onde a resistência dos parasitos à maioria dos grupos químicos é uma realidade (SANGSTER, 1999). Devido a estes fatores, estudos vêm sendo realizados visando ao desenvolvimento de alternativos para reduzir o uso de anti-helmínticos no controle da gastroenterite verminótica, como o uso de fungos nematófagos (SAUMELL, 1998) e o emprego de minerais como o cobre, por exemplo (WALLER, 1998).

O cobre é um microelemento ou elemento traço, componente essencial em dezenas de sistemas enzimáticos, responsável por processos como produção de energia, ação anti-radicais livres, formação da melanina e da elastina (MOURA, 1997; BRIAN, 1997). Além disso, interfere na produção de hemoglobina, formação dos ossos, pigmentação do pêlo e da lã e também está envolvido no funcionamento do coração e do sistema nervoso central (GEORGIEVSKII et al., 1982; CARTILHA DO AGRICULTOR, 1982; KOLB, 1987; SILVA e BARUSELLI, 2001). Também ajuda no desenvolvimento de anticorpos e replicação de linfócitos (MOURA, 1997). Assim, pesquisadores da Nova Zelândia relataram que a administração oral de 4,1 gramas de partículas de óxido de cobre (COWP) para ovinos, acarreta uma redução significativa no número de parasitas recuperados na necropsia, com uma redução de $96 \%$ para Haemonchus contortus e $56 \%$ para Ostertagia circumcincta (BANG et al., 1990). Alguns autores descrevem que há uma diferença de susceptibilidade de acordo com as espécies de helmintos. O H. contortus é mais sensível do que outros nematódeos, sugerindo que a administração de COWP, além de causar aumento da mortalidade deste parasito, também resulta na fecundidade reduzida das fêmeas sobreviventes no hospedeiro (BREMNER, 1961; CHARTIER et al., 2000). O COWP consiste em um núcleo central de cobre puro recoberto com uma mistura de óxido cuproso e cúprico. Depois da administração oral, as cápsulas de COWP são dissolvidas no rúmem e as partículas então passam para o abomaso, e se alojam nas dobras da mucosa. Neste ambiente ácido, o cobre é liberado durante um longo período de tempo, sendo a solubilidade dos íons inorgânicos dependente do pH (LANGLANDS et al., 1989; NYMAN, 2000). De acordo com BANG et al. (1990), a suplementação com cobre em ovinos resultou em benefícios no combate aos parasitas abomasais. Trabalhos realizados no RS demostraram a possibilidade de haver efeito dependendo da dose do cobre sobre os nematódeos do abomaso, sendo que a administração oral de 3,4 gramas de COWP foi mais efetiva para $\boldsymbol{O}$. circuncimcta enquanto a administração de 1,7 gramas COWP foi efetiva unicamente sobre o Trichostrongylus axei, não havendo efeito significativo sobre os nematódeos do intestino delgado. Os resultados mostraram que houve diferença significativa entre os grupos que receberam 3,4 gramas de COWP e o grupo controle com respeito a nematódeos abomasais, sugerindo que este microelemento pode ser incorporado como alternativa para a redução do uso de anti-helmínticos no controle da verminose ovina (NYMAN, 2000).

Tendo em vista a situação de resistência anti-helmíntica em ovinos, no RS, e a necessidade do desenvolvimento de métodos alternativos que possam 
contribuir com a redução do uso de anti-helmínticos, foi desenvolvido o presente trabalho com o objetivo de verificar o tempo de proteção do cobre contra reinfecções por helmintos gastrintestinais de ovinos em pastejo.

\section{MATERIAL E MÉTODOS}

Este trabalho foi realizado nos meses de fevereiro e março de 2000, na Embrapa Pecuária Sul, localizado no município de Bagé, RS, Brasil. A área experimental era composta de campo nativo com gramíneas, principalmente dos gêneros Aristida, Eragrostis, Melica, Paspalum, Piptochaetium e Stipa e leguminosas dos gêneros Desmodium, Rhynchosia, Trifollium e Stylosanthes.

Foram utilizados 32 ovinos da raça Corriedale, com aproximadamente 18 meses de idade, mantidos em campo nativo naturalmente contaminado por tricostrongilídeos e na lotação de $0,7 \mathrm{UA} / \mathrm{ha}$. Os animais experimentais foram identificados, pesados e dosificados, per os, com trichlorfon $100 \mathrm{mg} / \mathrm{kg}$ e ivermectin $0,2 \mathrm{mg} / \mathrm{kg}$. Após, foram alocados em quatro grupos experimentais: $\mathrm{T}_{\mathrm{I}}$ (tratado com cobre e necropsiado no 28-dia); $\mathrm{T}_{\text {II }}$ (sem cobre e necropsiado no 28 - dia); $\mathrm{T}_{\mathrm{III}}$ (tratado com cobre e necropsiado no 56ㅇia); $\mathrm{T}_{\mathrm{IV}}$ (sem cobre e necropsiado no 56ㅇia). Os grupos medicados com cobre receberam, via oral, uma cápsula gelatinosa de quatro gramas contendo 3,4 gramas de óxido de cobre. Os ovinos foram monitorados semanalmente para determinação do número de ovos por grama de fezes (OPG), volume globular (VG), aspartato aminotransferase (AST) e concentrações de cobre no plasma. Ao abate, foram determinados: carga parasitária (UENO e GONÇALVES, 1998), concentrações de cobre no fígado e peso dos fígados. Na análise estatística, as cargas parasitárias encontradas nas necropsias e as contagens de OPG foram transformadas em $\log _{10}($ contagem +1$)$ e submetidas à análise de variância e comparação múltipla de tratamentos (Newman Keuls Multiple Range Test), usando o programa computadorizado Animal Designs, G. Gettinby, University of Strathclyde, Glasgow, Escócia. Os demais dados foram submetidos ao mesmo procedimento sem as transformações logarítmicas. O VG foi transformado em raiz quadrada e submetido ao mesmo procedimento. Os dados de OPG, peso vivo e AST de todos os tratamentos foram analisados como quatro diferentes tratamentos até a necropsia realizada no $28^{\circ}$ dia experimental. Já os dados dos $\mathrm{T}_{\mathrm{III}} \mathrm{e} \mathrm{T}_{\mathrm{IV}}$ foram avaliados desde o início até o $56^{\circ}$ dia experimental. A concentração de cobre no fígado, peso e concentração de cobre no plasma foi também submetida à ANOVAe comparação múltipla de tratamentos (Newman Keuls Multiple Range Test), sem as transformações logarítmicas.

\section{RESULTADOS E DISCUSSÃO}

Os resultados demostraram que os níveis de cobre no plasma não tiveram alterações significativas $(\mathrm{P}>0,05)$ entre os diversos tratamentos. Quanto aos níveis de cobre detectados no fígado dos animais abatidos (Tabela 1), observou-se, que os lotes medicados com cobre, apresentaram níveis mais elevados que os não medicados $(\mathrm{P}<0,05)$, mas ainda dentro dos parâmetros considerados não tóxicos para a espécie ovina que seria de 800ppm (ORTOLANI, 1996). Outros fatores monitorados, que levam a inferir que o cobre, neste experimento, não atingiu níveis tóxicos, foram a ausência de diferenças significativas ( $\mathrm{P}>0,05)$ quanto ao volume globular e as concentrações de cobre no plasma. $\mathrm{O}$ peso dos fígados e peso vivo dos animais também não mostraram diferenças significativas (Tabela 1).

Em relação ao níveis de AST, os grupos tratados com cobre apresentaram durante todo o período experimental, valores levemente superiores aos grupos controles (Figura 1). Embora diferença significativa $(\mathrm{P}<0,05)$ tenha sido detectada apenas entre $T_{I}$ e $T_{\text {II. }}$ BANG et al. (1990) observaram que, em todos os grupos de ovinos tratados com 4,1 gramas de COWP, que a atividade no plasma de AST,

Tabela 1- Médias aritméticas e desvio padrão $( \pm d p)$ do peso dos fígados e das concentrações de cobre no fígado (ppm) dos animais tratados com cobre $\left(\mathrm{T}_{\mathrm{I}}\right.$ e $\left.\mathrm{T}_{\mathrm{III}}\right)$ e controles $\left(\mathrm{T}_{\text {II }}\right.$ e $\left.\mathrm{T}_{\mathrm{IV}}\right)$.

\begin{tabular}{ccc}
\hline Tratamentos & Peso dos fígados $(\mathbf{g})$ & Cobre $(\mathbf{p p m})$ \\
\hline $\mathbf{T}_{\mathbf{I}}$ & $368^{\mathrm{a}}$ & $557^{\mathrm{a}}$ \\
& $( \pm 129)$ & $( \pm 175)$ \\
& $390^{\mathrm{a}}$ & $312^{\mathrm{b}}$ \\
$\mathbf{T}_{\text {II }}$ & $( \pm 44)$ & $( \pm 130)$ \\
& $378^{\mathrm{a}}$ & \\
& $( \pm 127)$ & $425^{\mathrm{ab}}$ \\
$\mathbf{T}_{\text {III }}$ & $( \pm 240)$ \\
& $378^{\mathrm{a}}$ & $338^{\mathrm{b}}$ \\
& $( \pm 127)$ & $( \pm 95)$ \\
\hline
\end{tabular}

1- Animais dos $\mathrm{T}_{\mathrm{I}}$ e $\mathrm{T}_{\text {II }}$ necropsiados no $28^{0}$ dia e dos $\mathrm{T}_{\text {III }}$ e $\mathrm{T}_{\text {IV }}$ no $56^{\circ}$ dia experimental.

2- Médias seguidas de letras iguais na mesma coluna, não diferem entre si $(\mathrm{P}>0,05)$.

Ciência Rural, v. 34, n. 1, jan-fev, 2004. 


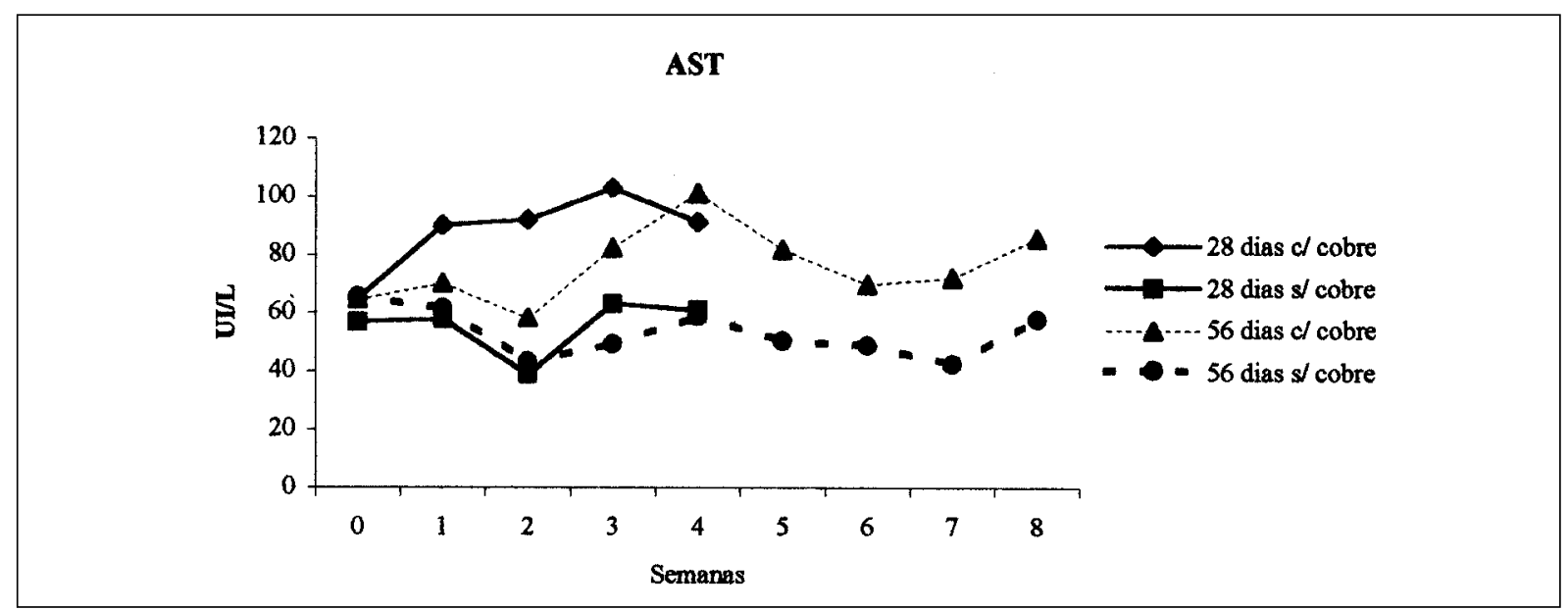

Figura 1 - Valores médios de aspartato aminotransferase (AST) dos animais tratados com cobre $\left(\mathrm{T}_{\mathrm{I}}\right.$ e $\left.\mathrm{T}_{\text {III }}\right)$ e controles $\left(\mathrm{T}_{\text {II }}\right.$ e $\left.\mathrm{T}_{\text {IV }}\right)$ durante o período experimental.

apresentava-se levemente aumentada nas amostras finais, porém, este aumento não causava efeitos colaterais como redução do peso ou danos no fígado.

A avaliação da evolução do OPG dos animais medicados com cobre $\left(\mathrm{T}_{\mathrm{I}}\right.$ e $\left.\mathrm{T}_{\mathrm{III}}\right)$ revelou que estes apresentaram menores contagens que a daqueles não medicados (Figura 2). Entretanto, na análise estatística, os dados mostraram que estas diferenças não eram significativas entre os grupos $(\mathrm{P}>0,05)$, devido à alta variabilidade nas contagens. $O$ percentual de redução de OPG do grupo $\mathrm{T}_{\mathrm{I}}$ com relação ao grupo $\mathrm{T}_{\text {II }}$ foi de $66 \%$ e do grupo $\mathrm{T}_{\text {III }}$ comparado ao $\mathrm{T}_{\text {IV }}$ de $81 \%$ quando avaliado ao final da quarta semana; já à oitava semana esta redução havia sido reduzida para $47,09 \%$. No presente estudo, com relação aos parasitos gastrintestinais, detectou-se que a administração de cobre contribuiu significativamente para uma menor reinfecção apenas pelo $\boldsymbol{H}$. contortus e somente nos animais abatidos na quarta semana após o tratamento, quando a redução foi de $60 \%$ nos medicados $(\mathrm{P}<0,05)$. Em relação aos demais helmintos gastrintestinais, as infeções foram baixas e com grande variabilidade tanto na necropsia do $28^{\circ}$ dia como no $56^{\circ}$ dia, o que inviabilizou conclusões mais precisas. Dentre os helmintos analisados, os dados mostram, claramente, uma predominância do $\boldsymbol{H}$. contortus sobre os demais parasitos (Tabela 2).

BREMNER (1961) sugeriu que a administração de COWP, além de causar aumento da mortalidade do $\boldsymbol{H}$. contortus, também resulta na fecundidade reduzida das fêmeas sobreviventes no hospedeiro. Neste estudo, as médias mostram que,

\section{OPG}

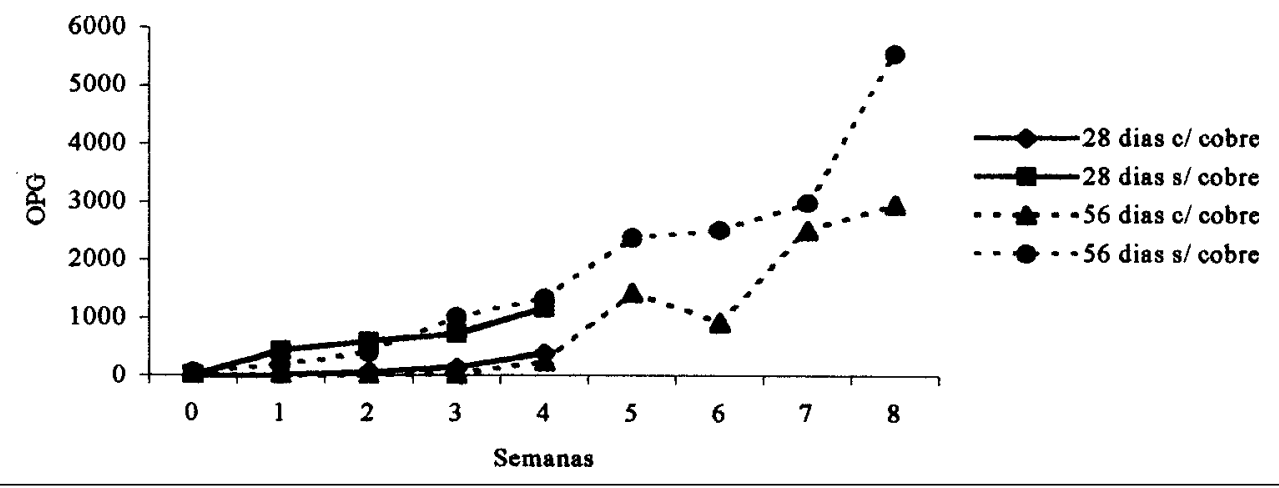

Figura 2 - Valores médios das contagens de ovos por grama de fezes (OPG) dos grupos tratados com cobre $\left(\mathrm{T}_{\mathrm{I}}\right.$ e $\left.\mathrm{T}_{\text {III }}\right)$ e controles $\left(\mathrm{T}_{\text {II }}\right.$ e $\left.\mathrm{T}_{\text {IV }}\right)$ durante o período experimental. 
Tabela 2 - Valores médios aritméticos e desvio padrão $( \pm \mathrm{dp})$ da carga parasitária recuperada na necropsia dos animais tratados com cobre ( $\mathrm{T}_{\mathrm{I}}$ e $\left.\mathrm{T}_{\text {III }}\right)$ e controles $\left(\mathrm{T}_{\text {II }}\right.$ e $\left.\mathrm{T}_{\text {IV }}\right)$.

\begin{tabular}{|c|c|c|c|c|c|c|c|c|c|}
\hline \multirow[b]{2}{*}{ Tratamentos } & & \multicolumn{8}{|c|}{ Helmintos } \\
\hline & & H. contortus & $\begin{array}{l}\text { Ostertagia } \\
\text { spp. }\end{array}$ & T. axei & $\begin{array}{c}T . \\
\text { colubriformis }\end{array}$ & $\begin{array}{l}\text { Cooperia } \\
\text { spp. }\end{array}$ & N. spathiger & $\begin{array}{l}\text { Trichuris } \\
\text { ovis }\end{array}$ & $\begin{array}{c}O . \\
\text { venulosum }\end{array}$ \\
\hline $\mathbf{T}_{\mathbf{I}}$ & $\begin{array}{l}\text { Médias } \\
\text { dp }\end{array}$ & $\begin{array}{c}334^{\mathrm{a}} \\
( \pm 187)\end{array}$ & $\begin{array}{c}6^{\mathrm{a}} \\
( \pm 9)\end{array}$ & $\begin{array}{c}0^{\mathrm{a}} \\
( \pm 0)\end{array}$ & $\begin{array}{c}36^{\mathrm{a}} \\
( \pm 76)\end{array}$ & $\begin{array}{c}14^{\mathrm{a}} \\
( \pm 25)\end{array}$ & $\begin{array}{c}0^{\mathrm{a}} \\
( \pm 0)\end{array}$ & $\begin{array}{c}0^{\mathrm{a}} \\
( \pm 0)\end{array}$ & $\begin{array}{c}1^{\mathrm{a}} \\
( \pm 1)\end{array}$ \\
\hline $\mathbf{T}_{\text {II }}$ & $\begin{array}{l}\text { Médias } \\
\text { dp }\end{array}$ & $\begin{array}{c}829^{\mathrm{b}} \\
( \pm 598)\end{array}$ & $\begin{array}{c}36^{\mathrm{a}} \\
( \pm 61)\end{array}$ & $\begin{array}{c}15^{\mathrm{a}} \\
( \pm 25)\end{array}$ & $\begin{array}{c}96^{\mathrm{ab}} \\
( \pm 116)\end{array}$ & $\begin{array}{c}10^{\mathrm{a}} \\
( \pm 14)\end{array}$ & $\begin{array}{c}0^{\mathrm{a}} \\
( \pm 0)\end{array}$ & $\begin{array}{c}0^{\mathrm{a}} \\
( \pm 0)\end{array}$ & $\begin{array}{c}2^{\mathrm{a}} \\
( \pm 3)\end{array}$ \\
\hline $\mathbf{T}_{\text {III }}$ & $\begin{array}{l}\text { Médias } \\
\text { dp }\end{array}$ & $\begin{array}{l}2050^{c} \\
( \pm 920)\end{array}$ & $\begin{array}{c}51^{\mathrm{b}} \\
( \pm 57)\end{array}$ & $\begin{array}{c}56^{\mathrm{a}} \\
( \pm 120)\end{array}$ & $\begin{array}{c}366^{\mathrm{ab}} \\
( \pm 621)\end{array}$ & $\begin{array}{c}16^{\mathrm{a}} \\
( \pm 19)\end{array}$ & $\begin{array}{c}10^{\mathrm{a}} \\
( \pm 19)\end{array}$ & $\begin{array}{c}0^{\mathrm{a}} \\
( \pm 1)\end{array}$ & $\begin{array}{c}15^{\mathrm{a}} \\
( \pm 12)\end{array}$ \\
\hline $\mathbf{T}_{\mathrm{IV}}$ & $\begin{array}{l}\text { Médias } \\
\text { dp }\end{array}$ & $\begin{array}{c}1753^{\mathrm{c}} \\
( \pm 1106)\end{array}$ & $\begin{array}{l}43^{\mathrm{ab}} \\
( \pm 31)\end{array}$ & $\begin{array}{l}21^{\mathrm{a}} \\
( \pm 29)\end{array}$ & $\begin{array}{c}344^{\mathrm{b}} \\
( \pm 353)\end{array}$ & $\begin{array}{c}33^{\mathrm{a}} \\
( \pm 38)\end{array}$ & $\begin{array}{c}6^{\mathrm{a}} \\
( \pm 11)\end{array}$ & $\begin{array}{c}2^{\mathrm{a}} \\
( \pm 4)\end{array}$ & $\begin{array}{l}10^{\mathrm{a}} \\
( \pm 8)\end{array}$ \\
\hline
\end{tabular}

1 - Animais dos $\mathrm{T}_{\mathrm{I}}$ e $\mathrm{T}_{\text {II }}$ necropsiados no $28^{0}$ dia e dos $\mathrm{T}_{\text {III }}$ e $\mathrm{T}_{\text {IV }}$ no $56^{0}$ dia experimental

2 - Médias seguidas de letras iguais na mesma coluna, não diferem entre si $(\mathrm{P}>0,05)$

com 56 dias, não houve uma contribuição significativa do uso do cobre como moderador no número de ovos. NYMAN (2000) observou um efeito do cobre na redução do OPG e contagem de parasitos recuperados no abomaso dos ovinos tratados com COWP, nas primeiras duas semanas após sua administração. Alguns autores testaram a eficácia do uso de óxido de cobre para o controle de parasitos nematódeos resistentes aos benzimidazois em cabras leiteiras; os resultados demonstraram uma eficácia de $75 \%$ na redução da carga de $\boldsymbol{H}$. contortus, assim como houve uma menor produção de ovos de $37-95 \%$, durante o estabelecimento de novas infecções por várias semanas (CHARTIER et al., 2000).

\section{CONCLUSÕES}

O uso de 3,4 gramas de óxido de cobre, para ovinos manejados extensivamente, não causa toxicidade e pode ser auxiliar no controle do $\boldsymbol{H}$. contortus, considerado o parasito de maior importância na região Sul do Brasil, protegendo contra reinfecções por até quatro semanas.

Ação do cobre em outros parasitos precisa ser determinada sob condições de maior desafio.

\section{AGRADECIMENTOS}

Os autores agradecem à Bayer do Brasil pelo financiamento das análises de concentrações de cobre no fígado e plasma, realizadas na Embrapa Gado de Corte e ao $\mathrm{CNPq}$ pela bolsa de formação de pesquisador II - Mestrado concendida à autora principal.

\section{REFERÊNCIAS BIBLIOGRÁFICAS}

BANG, K.S.; FAMINTON, A.S.; SYKES, A.R. Effect of copper oxide wire particle treatment on establishment of major gastrointestinal nematodes in lambs. Research in Veterinary Science, v.49, p.132-137, 1990.

BREMNER, K.C. The copper status of some helminth parasites, with particular reference to host-helminth relationships in the gastro-intestinal tract of cattle. Australian Journal of Agricultural Research, v.12, p.1188-1199, 1961.

BRIAN, B. Mineral nutrition in sheep. Agriculture and Rural representative, Gore bay, OMAFRA, 1997. Capturado em 14 maio. 2001. Online. Disponível em: http:// www.bireme.br.

CARTIlHA DO AGRICUlTOR. Os Animais: Publicação da Secretaria da agricultura. 2.ed. Porto Alegre : Corag, 1982. V.4, p.556.

CHARTIER S.C. et al. Efficacy of copper oxide needles for the control of nematode parasites in dairy goat. Veterinay Research Communications v.24, n.6, p.389-99, 2000.

ECHEVARRIA, F.A.M. Epidemiologia de nematódeos e o controle estratégico em ovinos lanados. In: PADILHA, T. Controle dos nematódeos gastrintestinais em ruminantes. Coronel Pacheco : EMBRAPA - CNPGL, 1996a. p.157-168.

ECHEVARRIA, F.A.M. Estratégias de controle da verminose em ovinos-como driblar o problema da resistência. In: SIMPÓSIO SOBRE CONTROLE DE PARASITOS, 1., 1996, Campinas. Anais... Campinas : CATI, 1996b. p.64-78.

ECHEVARRIA, F.A.M. et al. The prevalence of anthelmintic resistance in nematode parasites of sheep in Southern Latin

Ciência Rural, v. 34, n. 1, jan-fev, 2004. 
America: Brasil. Veterinary Parasitology,v.62, p.199-206, 1996.

GEORGIEVSKII, V.I.; ANNENKOV, B.N.; SAMOKHIN, V.I. Studies in the agricultural and food sciencies. Mineral Nutrition of animals. London : Butterworth, 1982. 475p.

KOLB, E. Fisiologia veterinária. 4.ed. Rio de Janeiro : Guanabara Koogan, 1987. 611p.

LANGLANDS, J.P. et al. Trace element nutrition of grazing ruminants. III으. Copper oxide powder as a copper supplement. Australian Journal Agricultutural Research, v.40, p.187-193, 1989.

MOURA, J.G.P. A Revolução dos nutrientes: um estudo sobre radicais livres, vitaminas, minerais e sua avaliação no exame do cabelo. Pelotas : Mundial, 1997. 265p.

NYMAN, H. Alternative methods of treating gastrointestinal nematodes in sheep, using Duddingtonia flagrans and copper wire particles. Uppsala, Sweeden : Swedish University of Agricultural Sciences, 2000. 54p. (Minor Field Studies. April, 2000. n.99).

ORTOLANI, E.L. Intoxicações e doenças metabólicas em ovinos: intoxicação cúprica, urolitíase e toxemia da prenhez. In: SILVA SOBRINO, A.G. Nutrição de ovinos. Jaboticabal: Funep,1996. 258p.
PADILHA, T.; MENDONZA, P.G. Controle microbiano de formas de vida livre dos nematódeos trichostrongilídeos: uma alternativa para a higienização das pastagens. In:

Controle dos nematódeos gastrintestinais em ruminantes. Coronel Pacheco : EMBRAPA-CNPGL, 1996. p.215-235.

SANGSTER, N.C. Anthelmintic resistance: past, present and future. International Journal for Parasitology, v.29, p.115-124, 1999.

SAUMELL, C.A. Colonização de bolos fecais bovinos e fezes ovinas por fungos nematófagos e sua ocorrência em fezes frescas bovinas nas Zona da Mata de Minas Gerais. 1998. 175f. Tese (Doutorado em Parasitologia) Curso de Pós-graduação em Parasitologia do Instituto de Ciência Biológicas, Universidade Federal de Minas Gerais.

SILVA, S.; BARUSELli, M.S. Os dez mandamentos da suplementação mineral. Guaíba : Agropecuária 2001. 106p.

UENO, H.; GONÇALVES, P.C. Manual para o diagnóstico das helmintoses de ruminantes. Salvador : Press Color, 1998. 143p.

WALLER, P.J. International approaches to the concept of integrated control of nematode parasites of livestock. International Journal for Parasitology, v.29, p.155-164, 1998. 